\title{
A formação de formadores e a integração do computador na licenciatura de matemática
}

\author{
Marceli Behm Goulart \\ Professora, UNICENTRO/Departamento de Matemática \\ behmgoulart@yahoo.com.br
}

\section{Maria Tereza Carneiro Soares}

Professora, UFPR/Departamento de Planejamento e Administração Escolar

mariteufpr@gmail.com

\section{Suely Scherer}

Professora, UFMS/Departamento de Matemática

susche@gmail.com

\section{Resumo}

$\mathrm{O}$ artigo apresenta resultados de uma pesquisa cujo objetivo foi identificar alguns elementos para uma proposta de formação de professores de licenciatura em Matemática para o uso das TIC. Os dados revelaram uma estreita relação entre o estágio que o professor se encontra no uso do computador e o domínio dos conhecimentos necessários para o uso deste recurso, e uma relação entre o estágio no uso do computador e o tipo de atividade desenvolvido nos processos de ensino e aprendizagem, relações estas que devem ser consideradas numa proposta de formação de professores de formadores.

Palavras chave: Computador. Formadores. Estágios. Conhecimentos. Matemática.

\section{Training the trainers and the computer integration in mathematics degree}

\begin{abstract}
This article presents the results from a qualitative research which goal was to define some elements for a proposal of teachers' training in the Math course to use the TICs. Data revealed a narrow relation between the teacher's level of computer familiarity and the mastering of necessary knowledge to use this tool and a relation between the computer familiarity and the kind of activity developed in the teaching and learning processes. Theses interfaces must be considered in a training proposal of training teachers.
\end{abstract}

Keywords: Computer. Teachers. Stages. Knowledge. Mathematics. 


\section{Introdução}

Após a publicação da Lei de Diretrizes e Bases da Educação Nacional (BRASIL, 1996), ora em vigor, foram aprovados pareceres e resoluções os quais deveriam nortear as propostas curriculares dos cursos de licenciatura em geral (BRASIL, 2001), bem como as diretrizes curriculares específicas para os cursos de licenciatura em Matemática (BRASIL, 2002). Estes documentos advertem sobre a necessidade de que o futuro professor adquira familiaridade com o computador como instrumento de trabalho, incentivando sua utilização para o ensino da matemática.

Mas é preciso ressaltar que, as mudanças curriculares, produzidas por estes documentos no interior das Licenciaturas em Matemática, não garantem que os futuros professores de Matemática, no curso de sua formação inicial, sejam plenamente preparados para integrar o computador nos processos de ensino e aprendizagem da escola básica. Isto se deve, entre outras questões, ao "poder pedagógico" do professor da licenciatura de transformar o currículo intencional num currículo implementado, sendo que ambos nem sempre coincidem.

Este fato fortalece a necessidade de que seja discutida e pesquisada a formação do professor universitário, quando da proposição de qualquer discussão sobre a formação de futuros professores, tema esse bastante negligenciado e pouco pesquisado (SZTAJN et al., 2005; EVEN, 2008).

O presente estudo é parte de uma pesquisa de doutorado (GOULART, 2009) que teve como objetivo identificar alguns elementos de uma proposta de formação de formadores de professores de Matemática para a integração do uso do computador em cursos de Licenciatura em Matemática. A hipótese inicial foi a de que docentes em diferentes estágios no uso do computador, têm necessidades de formação diferentes, isto é, domínio diferenciado dos conhecimentos para o uso do computador e fazem dele diferentes usos em processos de ensino e aprendizagem.

\section{Os estágios no uso do computador}

Segundo a UNESCO (2002a), planejar e definir estratégias para a integração do computador num programa de formação de professores exige uma avaliação e análise de vários elementos, dentre os quais, estão a identificação do estágio em que esses professores se encontram no uso da tecnologia e a identificação das suas necessidades de formação.

Dentre os modelos encontrados na literatura para a definição dos estágios no uso das Tecnologias da Informação e Comunicação (TICs) encontram-se os de Goos et al. (2003), Frota e Borges (2004), Assude (2007), UNESCO (2002b) e Hall e Hord (2006). Neste artigo, destaca-se o último, já que ele propõe um instrumento 
para a definição do estágio, adaptado e testado por Griffin e Christensen (1999), para analisar o estágio em que os professores se encontram no uso das TIC, e que segue descrito abaixo:

1) Não-usuário: foram identificados três estágios bem diferentes de não-usuários, como descritos abaixo:

a) Estágio de uso 0 - não - usuário: não mostra nenhum conhecimento do computador e nem mesmo se interessa em aprender sobre o assunto;

b) estágio de uso 1 - orientação: quando um professor age para aprender sobre o computador, ou exibe interesse em conhecer mais, sem contudo ter uma decisão de usá-lo;

c) estágio de uso 2 - preparação: o professor está preparando material e a si mesmo para o primeiro uso.

2) Usuários: nem todos professores seguirão necessariamente esta sequência.

d) Estágio de uso 3 - uso mecânico: é caracterizado pela experimentação do professor, que busca dominar o computador e, por isso, o trabalho de mudança acompanha mais as necessidades do professor do que as dos alunos, muitas vezes resultando num uso superficial;

e) estágio de uso $4 \mathrm{~A}$ - rotina: o professor já dominou o computador e seu uso e estabeleceu uma forma regular de trabalhar com ele; o uso está estabilizado;

f) estágio de uso 4B - refinamento: baseado nas suas reflexões e avaliações, eles fazem adaptações no uso que fazem do computador, a fim de aumentar os benefícios de seus alunos;

g) estágio de uso 5 - integração: o professor faz adaptações para o benefício de seus alunos, mas as faz em companhia de um ou mais professores;

h) estágio de uso 6 - renovação: o professor está explorando ou implementando alguns meios, para modificar o uso do computador de uma forma mais ampla, ou para substituir por outra tecnologia.

\section{As necessidades de formação para o uso do computador}

Além da identificação do estágio em que os professores se encontram no uso da tecnologia, outro elemento importante a ser considerado para o planejamento da integração do computador num programa de formação de professores é a avaliação e análise das necessidades de formação desses profissionais (UNESCO, 2002a).

A análise de necessidades, no âmbito particular das ações de formação de professores, deve ser capaz de produzir “objetivos válidos e fornecer informação 
útil para decidir sobre os conteúdos e as atividades de formação" (RODRIGUES, ESTEVES, 1993, p. 20).

Em relação aos diferentes usos do computador em processos de ensino e aprendizagem, Bruce e Levin (1997), apoiados em Dewey (1956) e buscando a centralidade dos usuários ou aprendizes, propuseram uma nova taxonomia, que segue descrita no Quadro 1.

Apesar de Kimmins e Bouldin (1996) terem proposto uma taxonomia específica para a Matemática, optou-se neste trabalho pela taxonomia proposta por Bruce e Levin (1997), porque a mesma é mais abrangente no que trata a utilização do computador nos processos de ensino e aprendizagem.

\section{QUADRO 1 - Taxonomia proposta por Bruce e Levin (1997)}

\begin{tabular}{|c|c|c|}
\hline Classe & Usos & Descrição \\
\hline \multirow{4}{*}{$\begin{array}{l}\text { MÍDIA } \\
\text { PARA } \\
\text { INVESTIGAÇÃO }\end{array}$} & $\begin{array}{l}\text { Construção de teoria } \\
(\mathrm{CTeo})\end{array}$ & $\begin{array}{l}\text { Tecnologia como mídia para pensar: } \\
\text { exploração de modelos e ferramentas de } \\
\text { simulação, softwares de visualização, } \\
\text { ambientes de realidade virtual, modelagem } \\
\text { de dados (definição de categorias, relações } \\
\text { e representações), modelos matemáticos, } \\
\text { integração de conhecimento. }\end{array}$ \\
\hline & Acesso a dados (Add) & Conexão ao mundo dos textos, vídeos e dados. \\
\hline & $\begin{array}{l}\text { Coleta de dados } \\
(\mathrm{CDd})\end{array}$ & $\begin{array}{l}\text { Uso da tecnologia para estender os sentidos: } \\
\text { registro de vídeo e som, sensores de } \\
\text { temperatura, movimento, batimento cardíaco. }\end{array}$ \\
\hline & $\begin{array}{l}\text { Análise de dados } \\
\text { (AnDd) }\end{array}$ & $\begin{array}{l}\text { Exploração da análise de dados, análise } \\
\text { estatística, ambientes para investigação. }\end{array}$ \\
\hline \multirow{4}{*}{$\begin{array}{l}\text { MÍDIA } \\
\text { PARA } \\
\text { COMUNICAÇÃO }\end{array}$} & $\begin{array}{l}\text { Preparação de } \\
\text { documentos (PrepD) }\end{array}$ & $\begin{array}{l}\text { Processadores de textos, apresentações gráficas, } \\
\text { expressões simbólicas. }\end{array}$ \\
\hline & $\begin{array}{l}\text { Comunicação } \\
\text { (Com) }\end{array}$ & $\begin{array}{l}\text { Comunicação com outros estudantes, } \\
\text { professores, especialistas em vários campos. }\end{array}$ \\
\hline & $\begin{array}{l}\text { Mídia colaborativa } \\
(\mathrm{MCol})\end{array}$ & $\begin{array}{l}\text { Ambientes de dados colaborativos, sistemas } \\
\text { suportes de decisão grupal, preparação de } \\
\text { documentos compartilhados. }\end{array}$ \\
\hline & $\begin{array}{l}\text { Mídia para ensino } \\
\text { (MEns) }\end{array}$ & $\begin{array}{l}\text { Sistemas tutoriais, simulações instrucionais, } \\
\text { exercícios e sistemas de prática. }\end{array}$ \\
\hline
\end{tabular}




\begin{tabular}{lll}
$\begin{array}{l}\text { MÍDIA } \\
\text { PARA } \\
\text { CONSTRUÇÃO }\end{array}$ & (MCons) & $\begin{array}{l}\text { Sistemas de controle (uso da tecnologia para } \\
\text { afetar o mundo físico), robótica, controle } \\
\text { de equipamento, construção de gráficos e } \\
\text { diagramas; }\end{array}$ \\
\hline MÍDIA & (MExp) & $\begin{array}{l}\text { Programas de desenho e pintura, composição } \\
\text { e edição de música, vídeos interativos e } \\
\text { PARA }\end{array}$ \\
EXPRESSÃO & multimídia,
\end{tabular}

Baseados nos trabalhos de Shulman (1986, 1987), Mishra e Koehler (2006) propõem um modelo que descreve os conhecimentos necessários aos professores para o uso das TICs, que é resultado da interação de três áreas de conhecimento: tecnologia, pedagogia e conteúdo específico:

1. Conhecimento do conteúdo específico (C): inclui conhecimento dos fatos centrais e conceitos do conhecimento (SHULMAN, 1986; 1987);

2. Conhecimento pedagógico $(\mathrm{P})$ : é o profundo conhecimento sobre os processos aprendizagem e práticas de ensino (SHULMAN, 1986; 1987);

3. Conhecimento pedagógico do conteúdo (PC): conhecimentos de pedagogia que são aplicáveis ao ensino do conteúdo específico (SHULMAN, 1986; 1987);

4. Conhecimento tecnológico (T): envolve as competências requeridas para operar com tecnologias específicas (MISHRA e KOEHLER, 2006);

5. Conhecimento tecnológico do conteúdo (TC): é o conhecimento sobre como a tecnologia pode modificar o conteúdo específico (MISHRA e KOEHLER, 2006);

6. Conhecimento pedagógico da tecnologia (PT): é a compreensão de como ensino e aprendizagem podem mudar a partir do uso de uma determinada tecnologia (MISHRA e KOEHLER, 2006);

7. Conhecimento tecnológico e pedagógico do conteúdo (TPC): é uma forma de conhecimento que vai além de todos os três componentes (conteúdo, pedagogia e tecnologia). TPC é a base de um bom ensino com tecnologia (MISHRA e KOEHLER, 2006).

\section{Metodologia da pesquisa}

A partir da hipótese de que formadores de professores, em diferentes estágios no uso do computador, evidenciariam diferentes conhecimentos e usos, realizou-se um estudo que teve como sujeitos 23 professores, que atuaram no primeiro e segundo 
semestres, de 2008, em cursos de Licenciatura em Matemática de seis universidades públicas do estado do Paraná.

Para atender aos propósitos deste estudo, foram selecionados dois instrumentos para a coleta de dados, quais sejam, questionário por ramificação e questionário.

O primeiro instrumento utilizado foi uma adaptação do questionário por ramificações proposto por Hall e Hord (2006) e que teve como objetivo a definição do estágio no uso do computador. Nessa questão utilizou-se a Escala de Likert e, portanto, o valor máximo a ser atingido poderia ser 5 e o valor mínimo 1.

O segundo instrumento foi subdividido em duas partes, sendo a primeira um conjunto de questões que teve como objetivo a avaliação do domínio dos diferentes conhecimentos pelos sujeitos da pesquisa e que se constitui numa adaptação do estudo de Archambault e Crippen (2009); e a segunda um conjunto de questões que buscou definir quais os usos que esses professores fazem do computador em processos de ensino e aprendizagem.

Com o intuito de identificar uma possível relação entre o estágio e o domínio dos conhecimentos para o uso do computador, utilizou-se elementos da estatística descritiva, a saber, a média aritmética dos escores atingidos em cada um dos conhecimentos por estágio.

Para investigar os usos mais frequentes do computador, em processos de ensino e aprendizagem em cada um dos estágios, buscou-se o apoio na Análise Hierárquica de Similaridade através do software CHIC (Classificação Hierárquica, Implicativa e Coesitiva).

A análise hierárquica de similaridade é um método de análise de dados estatísticos multidimensionais que permite "estudar e depois interpretar, em termos de tipologia e de semelhança (dessemelhança) decrescente, classes de variáveis constituídas significativamente a certos níveis de uma árvore de similaridade e se opondo a outros, nestes mesmos níveis" (ALMOULOUD, 2008, p. 306).

Em relação aos estágios os professores se autodefiniram como estando no estágio 2: 2 professores; estágio 3: 4 professores; estágio 4A: 2 professores; estágio 4B: 3 professores; e estágio 5: 10 professores. Como a frequência dos estágios 0 e 1 foi igual a um e do estágio 6 foi zero, decidiu-se unir os dois primeiros estágios em estágio 01 e desconsiderar o último.

$\mathrm{O}$ uso que os formadores, participantes da pesquisa, fazem do computador com seus alunos da Licenciatura em Matemática, conforme a taxonomia proposta por Bruce e Levin (1997) pode ser assim descrita: CTeo: 14 professores; Add: 14 professores; PrepD: 14 professores; Com: 10 professores; MEns: 6 professores; MExp: 5 professores; CDd: 3 professores; MCol: 1 professor; AnDd: 1 professor; 
MCons: nenhum professor; sendo que as variáveis que tiveram freqüência igual a zero ou 1 foram excluídas do estudo.

Dos 23 professores sujeitos desta pesquisa, 26,09\% declararam que não utilizam o computador em processos de ensino e aprendizagem no contexto da Licenciatura em Matemática, 39,13\% usam esporadicamente e 34,78\% disseram que usam frequentemente o computador com os alunos.

\section{Relação entre os estágios em que se encontram os professores formadores no uso do computador e os conhecimentos necessários para o uso das TIC em sala de aula}

Os valores médios dos escores em cada um dos conhecimentos estão dispostos em ordem decrescente na Tabela 1, que está acompanhado pelo respectivo estágio:

\section{TABELA 1 - Valor médio dos escores dos conhecimentos dos formadores de professores para o uso do computador, em ordem decrescente}

\begin{tabular}{|c|c|c|c|c|c|c|c|c|c|c|c|c|c|c|}
\hline & \multicolumn{2}{|c|}{ Conteúdo } & \multicolumn{2}{|c|}{ Pedagógico } & \multicolumn{2}{c|}{$\begin{array}{c}\text { Ped. do } \\
\text { Conteúdo }\end{array}$} & \multicolumn{2}{c|}{ Tecnológico } & \multicolumn{2}{c|}{$\begin{array}{c}\text { Ped. da } \\
\text { Tecnologia }\end{array}$} & \multicolumn{2}{|c|}{$\begin{array}{c}\text { Tecn. do } \\
\text { Conteúdo }\end{array}$} & $\begin{array}{c}\text { Tec. e Ped. do } \\
\text { Conteúdo }\end{array}$ \\
\hline $1^{\circ}$ & $4 \mathrm{~B}$ & 3,997 & $4 \mathrm{~B}$ & 4,113 & $4 \mathrm{~B}$ & 4,333 & $4 \mathrm{~B}$ & 4,22 & $4 \mathrm{~B}$ & 4,223 & $4 \mathrm{~B}$ & 4,557 & $4 \mathrm{~B}$ & 3,89 \\
\hline $2^{\circ}$ & 01 & 3,835 & $4 \mathrm{~A}$ & 3,665 & $4 \mathrm{~A}$ & 3,625 & $4 \mathrm{~A}$ & 3,0 & $4 \mathrm{~A}$ & 3,5 & $4 \mathrm{~A}$ & 3,5 & $4 \mathrm{~A}$ & 3,825 \\
\hline $3^{\circ}$ & 2 & 3,665 & 5 & 3,558 & 5 & 3,499 & 5 & 2,834 & 5 & 3,034 & 5 & 3,301 & 5 & 3,115 \\
\hline $4^{\circ}$ & 5 & 3,633 & 01 & 3,335 & 01 & 3,125 & 3 & 2,5 & 3 & 2,835 & 2 & 2,665 & 3 & 2,583 \\
\hline $5^{\circ}$ & 3 & 3,418 & 3 & 3,25 & 2 & 3,0 & 01 & 2,5 & 2 & 2,335 & 3 & 2,583 & 2 & 2,5 \\
\hline $6^{\circ}$ & $4 \mathrm{~A}$ & 3,17 & 2 & 3,0 & 3 & 2,895 & 2 & 2,335 & 01 & 2,335 & 01 & 2,165 & 01 & 2,33 \\
\hline
\end{tabular}

É possível observar que os professores dos estágios 01, 2, 3 e 5 evidenciaram ter maior segurança sobre os conhecimentos que não envolvem tecnologia (conhecimento do conteúdo, conhecimento pedagógico e conhecimento pedagógico do conteúdo), em comparação com os conhecimentos que envolvem o tecnológico, o pedagógico da tecnologia, o tecnológico do conteúdo, e o tecnológico e pedagógico do conteúdo, conhecimentos estes que qualquer professor mobiliza ou deveria mobilizar para o processo de ensino. Essa tendência não é observada em relação aos professores dos estágios 4A e 4B.

$\mathrm{Na}$ Tabela 1, é possível perceber que os estágios 01, 2 e 3 atingem as três últimas posições (menores escores) em todos os conhecimentos, exceto no conhecimento do conteúdo específico. Também são esses os estágios que atingem escores 
inferiores a 3, em todos os conhecimentos que envolvem a tecnologia, o que não acontece com nenhum outro estágio e em nenhum outro conhecimento, exceto no pedagógico do conteúdo em que o estágio 3 atinge o escore 2,895. Essa constatação levanta a hipótese de que o não uso (estágios 01 e 2) ou o uso não tão avançado (estágio 3) pode estar sendo influenciado não só pela falta de domínio dos conhecimentos tecnológicos, mas também pelo não domínio do conhecimento pedagógico e do conhecimento pedagógico do conteúdo, sendo, portanto, necessário que esses sejam abordados numa proposta de formação para o uso do computador em processos de ensino e aprendizagem para professores que estejam nesses estágios.

Em geral, em ordem decrescente dos valores dos escores em cada um dos tipos de conhecimentos, foi possível perceber a sequência: estágio 4B, 4A, 5, 3, 2 e 01 . Essa vizinhança aponta para uma diferença na ordem dos estágios propostos por Hall e Hord (2006), apesar de que os autores já afirmam que não há necessariamente uma linearidade no avanço nos estágios. Essa alteração pode ter suas justificativas na compreensão que os sujeitos desta pesquisa tiveram do que seja um trabalho colaborativo. Uma hipótese é a de que os formadores, depois de iniciado suas primeiras experiências com o uso do computador em sala de aula (Estágio 3), buscam a ajuda de colegas mais experientes para avançar no uso que fazem desse recurso e não um trabalho de trocas e decisões conjuntas, possivelmente compreendido pelos participantes desta pesquisa como estágio 5. Hipótese que exige investigações futuras.

\section{Relação entre os estágios no uso do computador em que os professores formadores se encontram e o seu uso efetivo}

Buscando conhecer as relações entre os estágios no uso do computador e os usos que os professores fazem do mesmo, o tratamento de dados com o CHIC forneceu uma árvore de similaridade que apresenta 2 classes de variáveis, conforme Figura 1.

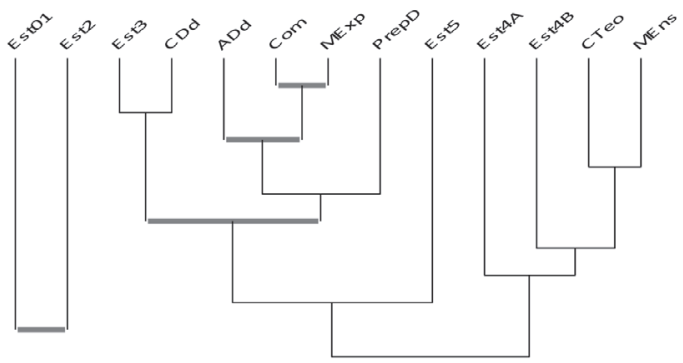

FIGURA 1. Árvore de similaridade gerada pelo software CHIC, como apresentação das relações entre estágios no uso do computador e tipos de uso. 
A primeira classe é constituída pelas variáveis estágio 01 (Est01) e estágio 2 (Est2), sendo caracterizada por formadores que não utilizam o computador com os seus alunos da Licenciatura em Matemática, e, portanto, não optaram por nenhum tipo de uso do computador.

A segunda classe comporta duas subclasses: a subclasse de variáveis (Est3, CDd, ADd, Com, MExp, PrepD e Est5) e a subclasse de variáveis (Est4A, Est4B, CTeo e MEns).

A primeira subclasse evidencia que os formadores de professores que estão no estágio 3 (Est3), utilizam o computador para a coleta de dados (CDd), para acesso a dados (ADd), para comunicação com os seus alunos (Com), como mídia de expressão (MExp) e para a preparação de documentos (PrepD). Esses usos também são característicos do estágio 5 (Est5), ou seja, de formadores que estão desenvolvendo um trabalho colaborativo com outros formadores, usos esses bastante concentrados na busca, registro, expressão e comunicação de ideias e conceitos, nas diferentes formas de linguagem.

A segunda subclasse revela que os formadores de professores que buscam fazer adaptações no uso do computador, para beneficiar a aprendizagem dos seus alunos, sem um trabalho colaborativo (Estágio 4B - Est4B), utilizam o computador para a construção de teoria e como mídia para ensino. O primeiro uso é bastante específico da área da Matemática, enquanto que o segundo precisa ser construído ou direcionado para essa área de conhecimento. Formadores que já estabeleceram uma forma regular de trabalhar com o computador também fazem esse mesmo uso do computador, sem, porém, buscar mudanças na forma de uso (Estágio 4A- Est4A).

Essa associação entre os estágios mais avançados (estágio 4A e 4B) e o uso mais relacionado ao ensino do conteúdo específico da Matemática (construção de teoria e mídia de ensino), pode ter sua causa na necessidade de que o formador seja capaz de articular, com bastante domínio, o conhecimento matemático e suas relações com os demais conhecimentos envolvidos no uso do computador: o pedagógico, o tecnológico e o pedagógico do conteúdo (MISHRA e KOEHLER, 2006). Esta articulação exigiria mais esforço dos formadores do que a utilização do computador para a busca, registro, expressão e comunicação de ideias e conceitos, nas diferentes formas de linguagem (típicos dos estágios 3 e 5). A possibilidade de utilizar o computador sem necessariamente exigir uma articulação com o conhecimento matemático, num primeiro momento, parece, de alguma forma, liberar o formador para se dedicar no domínio mais especificamente dos conhecimentos sobre a tecnologia e suas relações com o conhecimento pedagógico. Essa hipótese nos remete a novos questionamentos e novas pesquisas. 


\section{Discussões e considerações finais}

A análise dos dados aponta para a confirmação da hipótese de que os formadores de professores - professores da Licenciatura em Matemática - fazem diferentes usos do computador em sala de aula, ou seja, estão em diferentes estágios no uso do computador.

Pode-se também afirmar, que há uma relação entre os estágios no uso do computador e o domínio dos conhecimentos necessários para o seu uso em processos de ensino e aprendizagem, constatação esta que nos remete ao primeiro elemento a ser considerado em uma proposta de formação de formadores - professores da Licenciatura em Matemática - que vise a integração do computador nessa licenciatura, é o de que esta favoreça, não só a aprendizagem dos conhecimentos específicos da tecnologia, mas também os conhecimentos pedagógicos, conhecimentos pedagógicos do conteúdo, bem como todas as interrelações entre pedagogia, conteúdo específico e tecnologia.

O elemento apontado acima remete ao fato de que a formação dos professores formadores necessita ir muito além do estudo de softwares existentes, significa refletir e estudar sobre: o uso da tecnologia na mudança da dinâmica das aulas; as mudanças no papel do professor e do aluno; os processos de aprendizagem com o uso do computador (conhecimento pedagógico da tecnologia); as relações que se estabelecem entre a tecnologia e o conhecimento matemático (conhecimento tecnológico do conteúdo); e como se pode colocar em ação todos os conhecimentos envolvidos no uso do computador no ensino da Matemática, em sala de aula (conhecimento tecnológico e pedagógico do conhecimento).

Os dados também evidenciam um segundo elemento a ser considerado na formação de formadores de professores que é a organização de atividades, principalmente para formadores que se encontram nos estágios 01 e 2, que possibilitem a descoberta das potencialidades do computador no que se refere ao seu uso, em processos de ensino e aprendizagem da Matemática; e aos professores que se encontram nos estágios 3 e 5 a organização de atividades que contemplem experiências para o aprofundamento do uso do computador na construção do conhecimentos matemáticos propriamente dito por meio investigação, conjecturas, construção de hipóteses, criação de modelos, enfim, construção de conhecimento matemático.

A necessidade de que as propostas de formação de formadores de professores de Matemática considerem o estágio em que se encontram os formadores no uso do computador, os usos associados a cada um dos estágios e o domínio que os professores de cada estágio têm dos conhecimentos para o uso dessa tecnologia nos remete ao que Almeida (2004) denomina formação contextualizada de professores para ao uso pedagógico do computador, ou seja, que além de outras características, 
prevê que "as necessidades da formação emergem do contexto educacional no qual se busca desenvolver uma cultura que permita ao educador tornar-se um agente de mudança de sua própria atuação e de seu contexto" (p.85).

\section{Referências}

ALMEIDA, M. E. B. de, Inclusão digital do professor: formação e prática pedagógica. São Paulo: Editora Articulação, 2004.

ALMOULOUD, S. A. Análise e mapeamento estatístico de fenômenos didáticos com CHIC. In: OKADA, A. (Org.) Cartografia cognitiva: mapas do conhecimento para pesquisa, aprendizagem e formação docente. Cuiabá: KCM Editora, 2008, p. $303-324$.

ARCHAMBAULT, L.; CRIPPEN, K. Examining TPACK among K-12 online distance educators in the United States. Contemporary Issues in Technology and Teacher Education, v. 9, n.1, p.71-88, 2009.

ASSUDE, T. Teacher's practices and degree of ICT integration. In: European Research in Mathematics Education, 5, Larnaca. 2007. Disponível em: $<$ http:ermeweb. free.fr/CERME5b/>. Acesso em: 03/1/2009.

BRASIL. Lei n. 9394 de 20 de dezembro de 1996. Estabelece as diretrizes e bases da educação nacional. Diário Oficial [da] República Federativa do Brasil, Brasília, DF, 20 dez. 1996. Disponível em: < http://portal.mec.gov.br/seesp/arquivos/pdf/ lei9394_ldbn1.pdf >. Acesso em: 15/11/2007.

BRASIL. Resolução CNE/CP 1, 18 de fevereiro de 2002. Diretrizes para a Formação Inicial de Professores da Educação Básica, em Cursos de Nível Superior. Diário Oficial da União, Brasília, 9 de abril de 2002.

. Resolução CNE/CES 3, 18 de fevereiro de 2003. Diretrizes Curriculares para os Cursos de Matemática. Diário Oficial da União, Brasília, 25 de fevereiro de 2003.

BRUCE, B. C.; LEVIN, J. A. Educacional Technology: Media for Inquiry, Communication, Construction, and Expression. Journal of Educational Computing Research. v. 17, n. 1, 1997. p. $79-102$. 
DEWEY, J. The child and the curriculum and the school and society. Chicago: The University of Chicago Press, 1956.

EVEN, R. Learning to connect professional development for teachers and change initiatives in school mathematics. Symposium on the Occasion of the $\mathbf{1 0 0}^{\text {th }}$ Anniversary of ICMI. Roma, 2008. Disponível em: < www.unige.ch/math/EnsMath/ Rome2008/WG2/Papers/EVEN.pdf>. Acesso em: 16/01/2009.

FROTA, M. C. R; BORGES, O. Perfis de entendimento sobre o uso de tecnologias na Educação Matemática. In: Grupo de Trabalho 19 da Reunião Anual da Associação Nacional de Pós-Graduação e Pesquisa em Educação, 27, 2004, Caxambu. Anais... Disponível em: <http://paje.fe.usp.br/ anped/>. Acesso em: 19/06/2008.

GOOS, M. et al. Perspectives on technology mediated learning in secondary school mathematics classrooms. Journal of Mathematical Behavior. n. 22. 2003. p. 73 - 89. Disponível em: <www.elsevier.com/locate/jmathb>. Acesso em: 08/08/2007.

GOULART, M. B. A formação de formadores e a integração do computador na Licenciatura em Matemática. Curitiba, 2009. Tese (Doutorado em Educação) Setor de Educação, Universidade Federal do Paraná.

GRIFFIN, D; CHRISTENSEN, R. Concerns-Based Adoption Model (CBAM) Levels of Use of an Innovation (CBAM_LOU). Denton, Texas: Institute for the Integration of Technology into Teaching and Learning, 1999.

HALL, G. E.; HORD, S. M. Implementing Change: patterns, principles and potholes. 2. ed. Boston: Pearson Education, 2006. 304 p.

KIMMINS, D.; BOULDIN, E. Making Mathematics Come Alive with Technology. 1996. Disponível: <http://frank.mtsu.edu/ itconf/papers96/kimmins.html>. Acesso em: 28/11/2007.

MISHRA, P.; KOELHLER, M. J. Technological pedagogical content knowledge: a framework for teacher knowledge. Teachers College Record, v.108, n. 6, p. 1017-1054, jun. 2006.

RODRIGUES, A.; ESTEVES, M. A análise de necessidades na formação de professores. Porto: Porto Editora, 1993. 
SBEM. Subsídios para a discussão de propostas para os cursos de Licenciatura em Matemática: uma contribuição da Sociedade Brasileira de Educação Matemática. 2003. Disponível em: <www.sbem.com.br >. Acesso em: 28/05/2007.

SHULMAN, L. Those Who Understand: knowledge growth in teaching. Educational Research. v. 12, n. 2, p. 4 - 14, 1986.

. Knowledge an Teaching: foundations of the new reform. Harvard Educational Review. v. 51, n.1, p. 1- 22, fev. 1987.

SZTAJN, P. et al. And who teaches the Mathematics teachers? Professional Development of teacher developers. ICMI 15, Lindóia, 2005. Disponível em: $<$ stwww.weizmann.ac.il/G-math/ICMI/sztajnCPTM_ICMI_prop.doc>. Acesso em: 16/01/2009.

UNESCO - United Nations Educational, Scientific and Cultural Organization. Information and Communication Technologies in Teacher Education: a planning guide. Paris. 2002 (a). Disponível em: < http://unesdoc.unesco.org/ images/0012/001295/129533e.pdf > . Acesso em: 05/12/2007.

Information and Comunication Technology: a curriculum for schools and programme of teacher development. Paris. 2002 (b). Disponível em: < http:// unesdoc.unesco.org/images/0012/001295/129538e.pdf > . Acesso: 28/11/2007.

Submetido em maio de 2011

Aprovado em março de 2012 